\title{
Advanced UV Absorbers for the Protection of Human Skin
}

\author{
Dietmar Hüglin* \\ KGF-SCS Industrial Investigator Award 2015
}

\begin{abstract}
The increasing awareness of the damaging effects of UV radiation to human skin triggered the market introduction of new cosmetic UV absorbers. This article summarizes the outcome of a multi-year research program, in which the author contributed to the development of different new UV filters. First of all, the molecular design and the basic properties of bis-ethylhexyloxyphenol methoxyphenyl triazine (BEMT) will be presented. This oil-soluble filter, which today is widely used in both beach products and skin care products, exhibits inherent photostability and strong broad-spectrum UV-A+B absorbance. Based on the concept of micronized organic UV absorbers, the UV-B filter tris biphenyl triazine (TBPT) will be introduced. At present TBPT exhibits the highest efficacy of all cosmetic UV absorbers in the market (measured by area under the UV spectrum). Finally, the concept of liposomogenic UV absorbers will be featured. This approach was developed to create waterresistant UV filters, as liposomogenic structures are thought to integrate into the lipids of the horny layer. Due to prohibitively high costs, this technology did not result in a commercial product so far.
\end{abstract}

Keywords: Cosmetics · Liposome · Photostability · Sun protection · UV absorbers · Water resistance

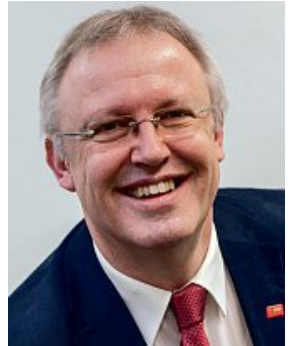

Dietmar Hüglin joined Ciba-Geigy in Basel in 1990 after completing his PhD in Germany (University Freiburg i.Br., Prof. H. Zimmermann). He has been working as a research chemist in agrochemicals, electronic materials and cosmetic UV absorbers and since 2012 at BASF Schweiz $A G$ as Vice President Research (Executive Expert). He holds nearly 80 patents and publications.

\section{Introduction}

The presence of ultraviolet (UV) absorbers in skincare and cosmetic products represents a key benefit that the cosmetics industry can provide to consumers. Increasing awareness of the damaging effects of UV radiation has led to a significant demand for improved protection of human skin, the largest organ of the body. Today very efficient protection can be achieved by topically applied UV absorbers, which are part of two main classes of cosmetic products: beach products (for sun care, protecting against sunburn, also called UV erythema) and skin care products (for day care, delaying photo-induced aging of skin).[1] Over the last 25 years, the author has been a member of an R\&D team with the objective to discover and develop new cosmetic UV absorbers and define new standards. This article aims to summarize some of the main outcomes of the research program for advanced UV absorbers for the protection of human skin. ${ }^{[2,3]}$

\subsection{Why Do We Need UV-B and UV-A Protection?}

UV radiation, which causes damage to skin, is divided into two regions: UV-B (290-320 nm) and UV-A (320-400 nm). UV-A is divided into UV-A1 (340-400 $\mathrm{nm})$ and UV-A2 (320-340 nm). While UV-B radiation is the main cause for photo-induced erythema, skin aging and skin cancer, UV-A (besides contributing to erythema formation and skin cancer) penetrates deeper into human skin and damages the collagen structure. This is the main reason for the observation that UV-A also contributes to premature skin aging. In order to achieve an optimal protection from detrimental effects, sunscreens contain a blend of different UV absorbers with different spectral characteristics. The sun protection factor (SPF) is a measure for the overall UV absorbing performance of sunscreens.

\subsection{Main Requirements for Cosmetic UV Absorbers}

There are two classes of cosmetic UV absorbers: the majority of the filters are organic molecules with a UV chromophore, which are incorporated into the oil-phase of sunscreen emulsions (soluble UV absorbers). These filters must exhibit good solubility in cosmetic oils. On the other hand, micro fine particles of inorganic materials like $\mathrm{TiO}_{2}$ have also an important role in the market (insoluble UV absorbers).

Cosmetic UV absorbers should exhibit a strong spectral absorbance (efficacy) in the relevant UV-region. The UV-absorbing molecule should be photostable under the application conditions and must not form photo-products upon irradiation, reducing the efficacy of the sunscreen. Moreover, in order to provide water-resistance and maintaining protection if exposed to an aquatic environment, the UV absorber should not be easily washed away from the skin surface by sweat or during swimming. 


\subsection{Anticipating Future Needs - Research Directions for New UV Absorbers}

Towards the end of the 1980s, consumers became increasingly aware of the harmful effects of sun, triggered by reports from dermatologists in newspapers, magazines and TV about the potential risk of skin cancer induced by UV exposure. The function of sunscreens started moving from mere protection against sunburn to skin health prophylaxis. With this trend in mind producers of cosmetic sunscreen products approached the chemical industry and expressed their fundamental unmet needs for better UV absorbers:

i) Inherently photostable UV-A absorbers. The objective was to overcome the main limitation of the commonly used UV-A absorber butyl methoxy dibenzoylmethane (BMDBM), which is instable when irradiated with UV light, if not protected by a stabilizer. The new UV filter should be efficient in use, exhibiting constant efficacy, without loss of extinction upon irradiation. Thus, the amount of such filter used for a certain protection level is expected to be less as compared to an unstable system. Moreover, with an inherently photostable UV-A absorber there should be no need to worry about the toxicology of possible photoproducts.

ii) UV absorbers with high efficacy in the $\boldsymbol{U V}-\boldsymbol{B} / \boldsymbol{U V}-\boldsymbol{A 2}$. The objective was to develop a filter for maximal UV protection (SPF) at lowest concentration in use.

iii) Inherently water-resistant $U V$ absorbers. The objective was to provide a maximum of wash-fastness on the molecular level. These UV absorbers should eliminate the need to frequently reapply the sunscreen in order to prevent sunburn and other damage to skin. Moreover, they should support the growing trend of outdoor sport activities in which the sunscreen layer is diminished by water from the outside (swimming) or from the inside (sweating).

In the course of the research program many different routes were taken to reach the targets. Cosmetic UV absorbers are strongly regulated and their approval for use requires numerous human toxicological and ecotoxicological studies, before being registered and commercialized. Therefore, the risk of failure is high, even when all technical and market objectives are met (e.g. prohibitive production cost).

\subsection{Technology Concepts}

Addressing unmet need i): Oil-soluble UV-A and UV-A+B (broad-spectrum) absorbers based on hydroxy-phenyl-triazine
(HPT) chemistry. This approach finally resulted in the commercial product bis-ethylhexyloxyphenol methoxyphenyl triazine (BEMT), the leading UV broad-spectrum filter on the market (Tinosorb ${ }^{\oplus} \mathrm{S}$ ).

Addressing unmet need ii): Insoluble (micronized) organic UV absorbers based on different chemistries. As current insoluble filters are inorganic minerals, this concept is new. Up to now, two products have entered the cosmetics market (Tinosorb ${ }^{\circledR} \mathrm{M}$, Tinosorb ${ }^{\circledR} \mathrm{A} 2 \mathrm{~B}$ ).

Addressing unmet need iii): a) Liposomogenic UV absorbers. This concept was developed to create water-resistant sunscreens, as liposomogenic structures are thought to integrate into the lipids of the stratum corneum (horny layer). Different lead candidates were obtained based on inherently liposomogenic molecules containing UV chromophores. Due to several reasons, e.g. high cost of synthesis and purification, this approach did not lead to commercial products. b) Reactive UV absorbers, which change their chemical structure after application on skin or covalently bind to native structures of skin, leading to enhanced performance (e.g. water resistance). After thorough assessment of risks related to human safety, this approach was not further pursued and will not be described in this article.

Sunscreen manufacturers have five basic requirements for UV absorbers: efficacy, safety, registration, patent freedom and cost in use. All of these must be fulfilled by new filters before they can be incorporated in a final product. Due to demanding and unpredictable tox and ecotox studies, combined with a time-consuming registration process, typically 10 years are required from product idea to commercialization. The process and its duration is comparable to the development of a new active pharmaceutical ingredient. ${ }^{[2]}$

\section{Case Studies}

\subsection{Bis-Ethylhexyloxyphenol Methoxyphenyl Triazine}

Polymeric substrates like automotive coatings and plastics, used in outdoor applications, strongly suffer from UVinduced photodegradation, if not protected by light stabilizers. In order to provide stability over many years the UV absorber itself must be inherently photostable and should not be subject to photo-chemical degradation processes. During the last 60 years continuous advancement has been achieved by developing stabilizers with higher efficacies and photostabilities, moving from benzophenones to benzotriazoles and, lately, to the most effective class, hydroxyphenyltriazines (HPT). ${ }^{[4,5]}$ The general chemical structure of HPTs,

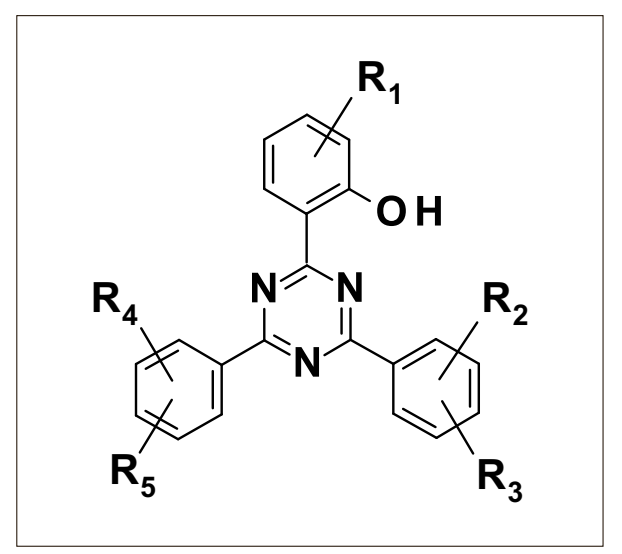

Fig. 1. Generic structure of HPTs with at least one ortho-OH group.

bearing at least one ortho $\mathrm{OH}$-group at the phenyl moiety, is depicted in Fig. 1.

If at least one $\mathrm{OH}$-group is present in the ortho position of the phenyl moiety, all three classes are capable of efficiently transforming UV radiation into thermal energy by a process called phototautomerism: absorption of UV radiation leads to singlet state excitation, followed by fast internal conversion. Enforced by the intramolecular hydrogen bond, the $\mathrm{H}$-atom undergoes a fast tautomeric transfer to the adjacent $\mathrm{O}$-atom (benzophenone) or $\mathrm{N}$-atom (benzotriazol, triazine), releasing the absorbed UV energy through vibronic relaxation as harmless thermal energy. In the last step of the photophysical cycle the proton moves back to its original position. The whole proton transfer cycle lasts less than $1 \mathrm{ps}\left(10^{-12} \mathrm{~s}\right)$. Since it is much faster than other competing processes, which may occur after excitation and lead to photo-decomposition, it is a very efficient mechanism for energy dissipation. ${ }^{[3,6]}$

In the following study, the molecular design of bis-ethylhexyloxyphenol methoxyphenyl triazine (BEMT, trade name: Tinosorb ${ }^{\circledR}$ S, BASF) will be presented.

Hydroxyphenyltriazine (HPT) was chosen as preferred chemistry because of its significantly higher molecular absorption coefficient for UV-A $(\varepsilon=45000$ $\left.75000 \mathrm{M}^{-1} \mathrm{~cm}^{-1}\right)$ as compared to benzotriazoles $\left(\varepsilon=15000-30000 \mathrm{M}^{-1} \mathrm{~cm}^{-1}\right)$. More than 500 different HPTs were prepared, exhibiting different $\mathrm{OH}$-patterns (number and location of $\mathrm{OH}$-groups at the three phenyl moieties) and different alkyl-/ alkoxy-substituents. As the HPT moiety without further substituents is not soluble in cosmetic oils and acts like a UV pigment, lipophilic substituents are required for good oil solubility. Fig. 2 depicts the spectral performance of different HPTs with selected $\mathrm{OH}$-patterns in the chromophore. ${ }^{[3,7]}$

The strong absorption of tri-phenyltriazines shown in the UV-B range (see Fig. 2 , case A) has $\pi \pi^{*}$ character. As an ortho- 


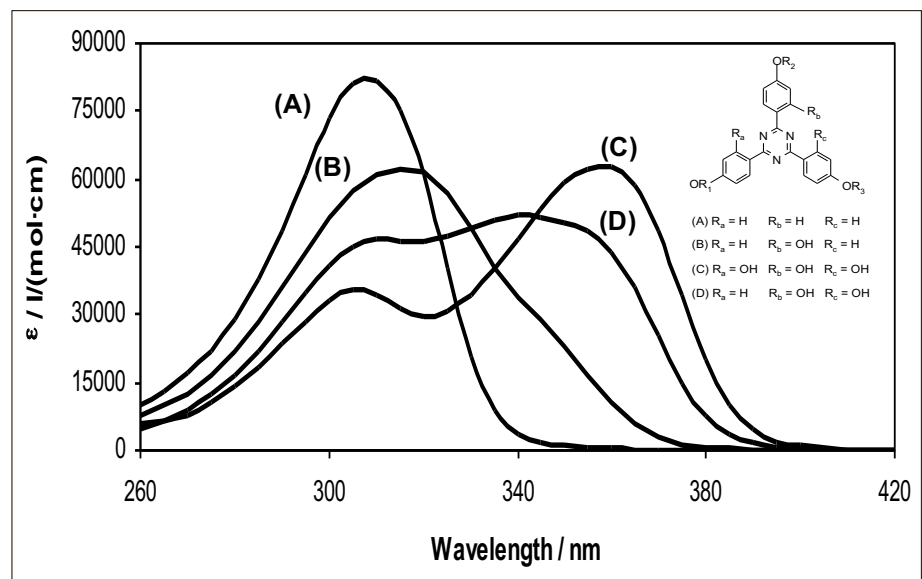

Fig. 2. UV spectral performance of HPT derivatives as function of substitution; $R_{1}$ $=$ methyl, $R_{2}$ and $R_{3}=$ 2-ethyl-hexyl.
BEMT is prepared in three chemical steps (Scheme 1): Grignard reaction of 4-bromo-anisole with cyanuric chloride (step 1), followed by Friedel-Crafts acylation of the bis-chloro-triazine with two equivalents of resorcinol (step 2). The final product BEMT is obtained by alkylation of the two para-OH groups with 2-ethylhexyl-chloride (step 3).

Today BEMT is one of the largest volume specialty chemicals synthesized by Grignard reaction. hydroxyl group is introduced (Fig. 2, case $\mathrm{B})$, a UV-A band emerges, which is caused by an intramolecular charge transfer $\left(\pi \pi^{*}\right)$. With two ortho-hydroxyl groups at different phenyl moieties, this UV-A absorption significantly increases (Fig. 2, case D) and with three, even more so (Fig. 2, case C). The optimized broad-spectrum spectrum UV-A+B was obtained with case D (Fig. 2) referring to BEMT, which exhibits $a b$ sorption maxima in the UV-B $(310 \mathrm{~nm}, \varepsilon$ $\left.=46800 \mathrm{M}^{-1} \mathrm{~cm}^{-1}\right)$ and UV-A $(343 \mathrm{~nm}$, $\left.\varepsilon=51900 \mathrm{M}^{-1} \mathrm{~cm}^{-1}\right)$, respectively. Fig. 3 shows the $E(1,1)$ spectrum and chemical structure of BEMT.

Due to the presence of two intramolecular hydrogen bonds, which can undergo photo-tautomerism upon excitation, this class exhibits a very high inherent photostability. Within the range of experimental error, BEMT remains stable after 10 hours of simulated solar irradiation, whereas BMDBM is degraded (Fig. 4). ${ }^{[3]}$

Having chosen the ideal chromophore, oil solubility had to be provided via suitable lipophilic substituents. Numerous efforts with different alkyl- and alkoxy patterns were made in order to move from research sample to product candidate. This step proved to be difficult, because of the limiting fact that the product had to be isolated in very high purity (>98\%). However, purification by chromatography is not feasible for cosmetic UV absorbers, due to the high cost for industrial production. Typical HPT research compounds, which were isolated by crystallization, exhibited melting points above $100{ }^{\circ} \mathrm{C}$ and were insoluble in cosmetic oils. On the contrary, research compounds which were lipophilic enough to provide sufficient oil solubility did not crystallize. They were isolated as highly viscous oils and only chromatography was applicable for purification.

Good oil solubility of chromophore D was obtained with R1 = methyl and R2 = R3 = 2-ethyl-hexyl. The resulting molecule BEMT exhibits crystallinity at room temperature (melting point $\mathrm{ca} .80^{\circ} \mathrm{C}$ ) and is inherently water-resistant (Fig. 5).

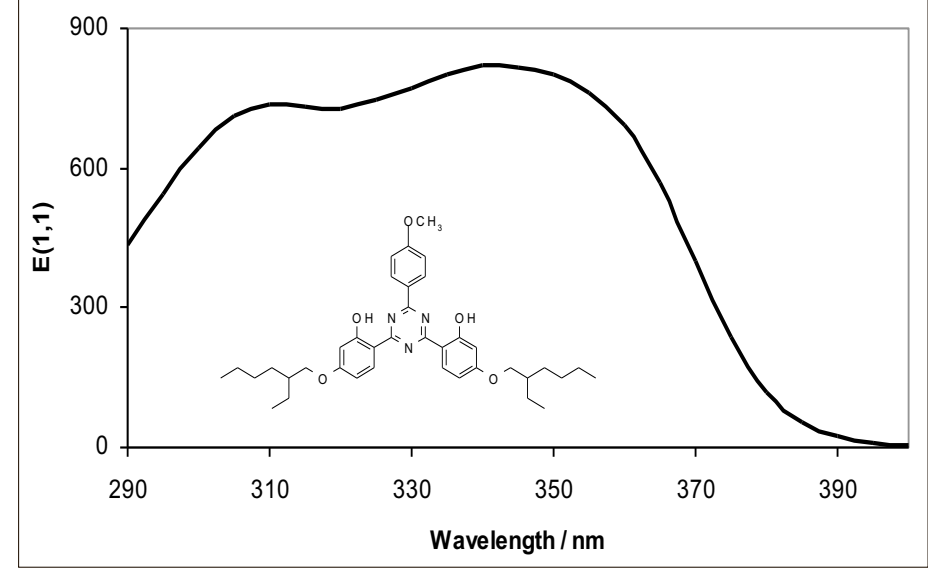

Fig. 3. UV spectrum (in ethanol) and chemical structure of bis-ethylhexyloxyphenol methoxyphenyl triazine (BEMT); E $(1,1)$ is the extinction at $1 \%$ concentration and $1 \mathrm{~cm}$ optical pathlength.

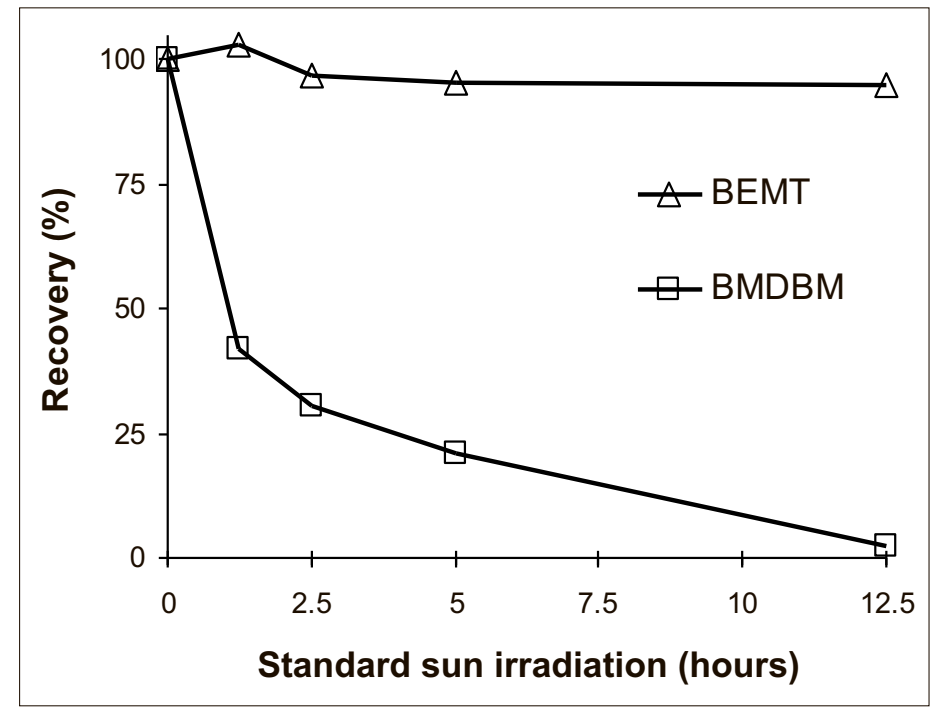

Fig. 4. Recovery of BEMT and BMDBM after simulated solar irradiation.

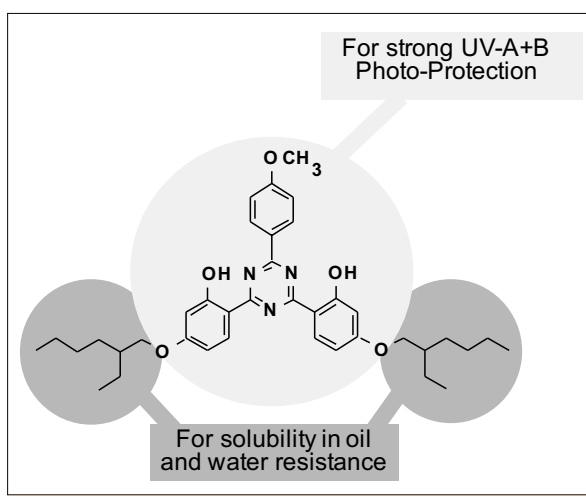

Fig. 5. Molecular design of BEMT for absorption efficacy, oil solubility and water resistance.

\subsection{Tris Biphenyl Triazine (TBPT) - a Highly Efficient Particulate Organic UV Absorber}

In the development of new UV absorbers, insolubility of the active substance can be a severe problem. ${ }^{[2]}$ Making a virtue of necessity, the concept of particulate organic UV filters has been invented. UV filters with extremely low solubility in oil and in water can be micronized in an aqueous phase employing a wet-milling process. This has been applied to methylene bis-benzotrazolyl tetramethyl-butylphenol (MBBT) $^{[2,3]}$ and recently also to tris-biphenyl triazine (TBPT). The latter was ap- 


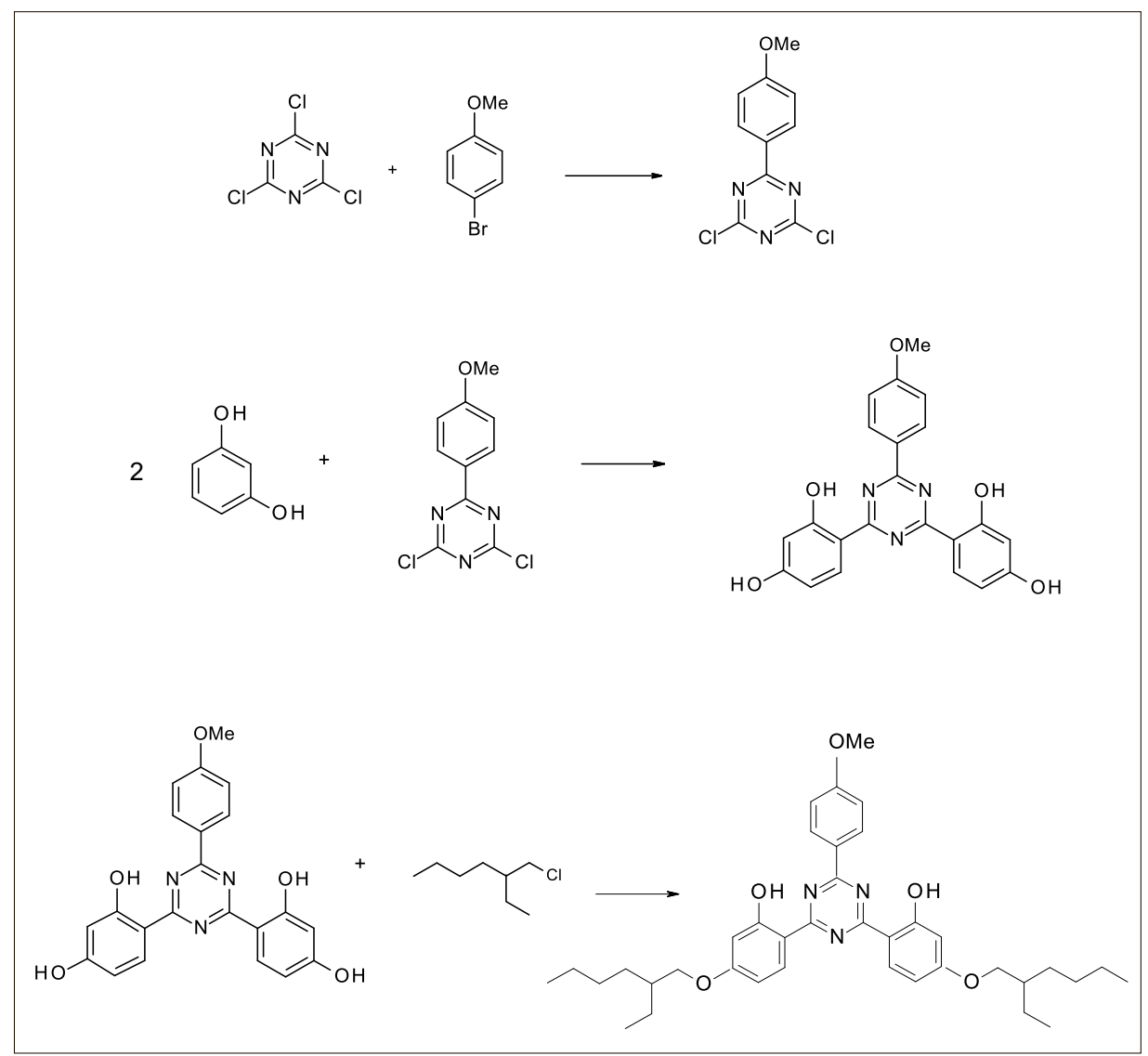

Scheme 1. Synthesis of BEMT.

proved for use in sunscreens in Europe in 2014. ${ }^{[8]}$ The pure substance is a solid with a melting point at $261^{\circ} \mathrm{C}$.

The structure of the active substance and a transmission electron microscopic picture of the final product is shown in Fig. 6 . Considering the average particle size of TBPT in the formulation, Tinosorb ${ }^{\circledR} \mathrm{A} 2 \mathrm{~B}$ is labeled as a nano-product.

UV/VIS spectra of the particle dispersions were measured using a UV/VIS spectrometer with an integrating sphere accessory. ${ }^{[9]}$ Although the solubility of TBPT in most organic solvents is very low, the solubility in dioxane is sufficient to measure a UV spectrum. In Fig. 7 the specific extinction $\mathrm{E}(1,1)$ of TBPT is shown when dissolved in dioxane and as a particle dispersion with the final particle size of the product at the end of the milling process.

The electronic transition reflected in the absorption spectrum of the compound dissolved in dioxane has strong intensity and is of $\pi \rightarrow \pi^{*}$ nature. ${ }^{[10]}$ The maximum of the absorption curve occurs at a wavelength of $315 \mathrm{~nm}$. The spectrum of the aqueous dispersion shows a shoulder at about $340 \mathrm{~nm}$, which is caused by intermolecular interactions of the $\pi$-electrons of the chromophores stacked inside the particles.

Fig. 8 shows the spectrum of particulate TBPT at different particle sizes. It is evident from these results that the efficacy of the UV absorbance strongly increases with decreasing particle size. The same phenomenon had also been observed with particulate MBBT. $[2,3]$

The assessment of photostability was conducted on quartz plates, using an Atlas CPS+ solar simulator. After irradiation, the samples were analyzed quantitatively by HPLC. [11]

Fig. 9 shows the recovery of the parent molecule TBPT, as function of standard sun irradiation time. The results demonstrate photostability of TBPT under such conditions, although the molecule contains not a single ortho-OH group. This can be explained by the presence of large aryl groups, which facilitate the process of internal conversion. ${ }^{[10]}$

\subsection{Liposomogenic UV Absorbers}

Amphiphilic molecules with structural components like phospholipids, if dispersed in water, can form spherical vesicles with bilayer structures, called liposomes (Fig. 10).

Liposomogenic molecules are thought to integrate into stratum corneum lipids, thus being retained in the skin and providing water-resistance on a molecular level. Molecular design concepts for chromophore-containing bilayer forming amphiphiles have already been described. ${ }^{[12]}$ In ref. [13] several structures are reported with UV absorbing chromophores and liposome-forming properties. One of these molecules designated 101a (Fig. 11) will be discussed in more detail. The synthesis of 101a is described in example 1 in ref. [13].

Fig. 6. Structure of the active substance (TBPT) and electron microscopic picture of the final product dispersion of Tinosorb ${ }^{\circledR} \mathrm{A} 2 \mathrm{~B}$.

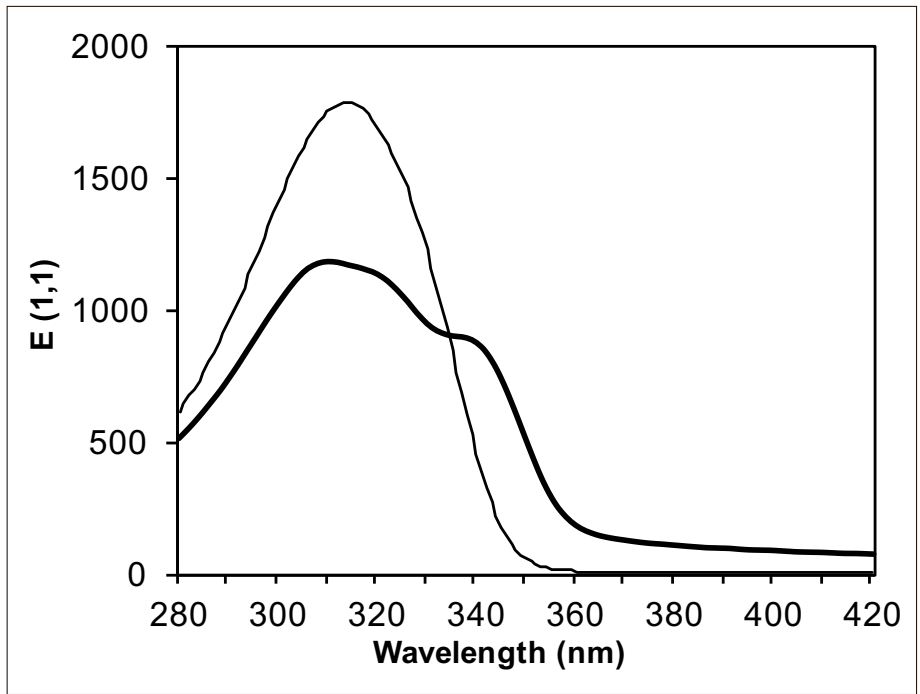

Fig. 7. TBPT dissolved in dioxane (thin line) and particulate TBPT in aqueous dispersion (thick line). $d_{0.5}=105 \mathrm{~nm}$ (average particle size, volume weighted). At present, this UV filter exhibits the highest efficacy of all commercial products (measured by area under the UV spectrum). 

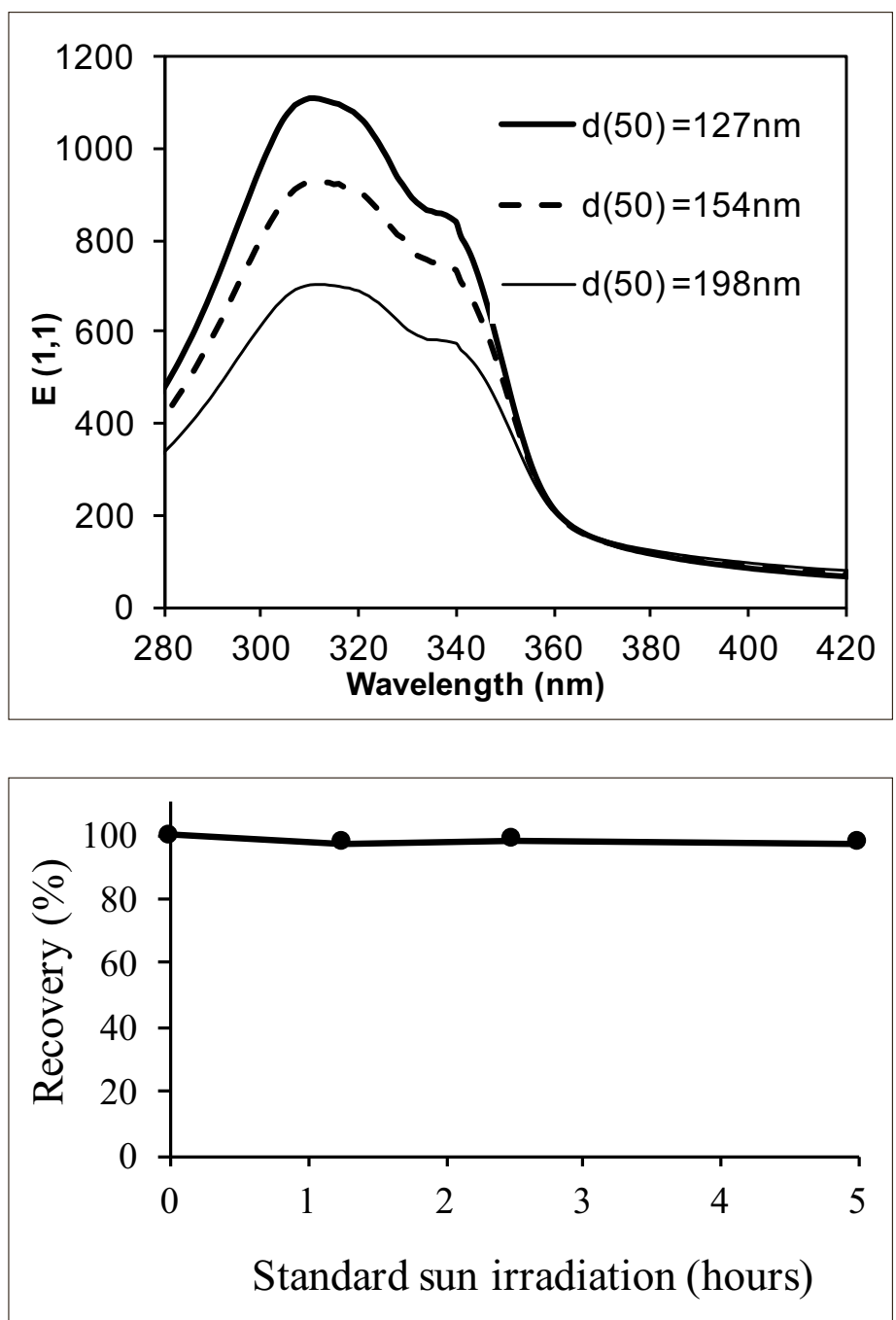

Fig. 8. Particulate TBPT at different particle sizes given as $\mathrm{d}_{0.5}$ (volume weighted).

Fig. 9. Recovery of TBPT after simulated solar irradiation.
The ratios of the concentration in the pig skin after desorption and adsorption represent the water resistance. It was demonstrated that the liposomogenic UV absorber 101a exhibits much higher water resistance than the conventional water-soluble product PBSA. The results are summarized in Table 1.

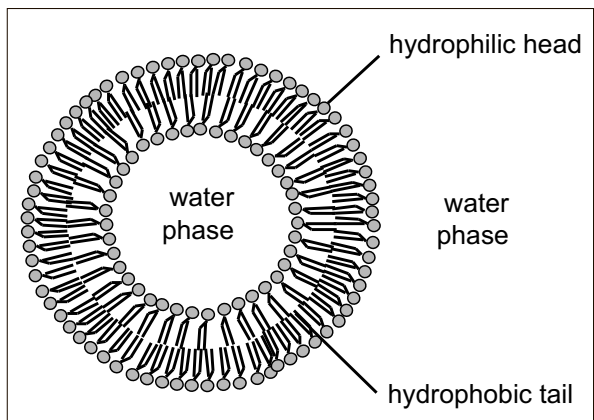

Fig. 10. Generic structure of a liposome, consisting of liposomogenic molecules.

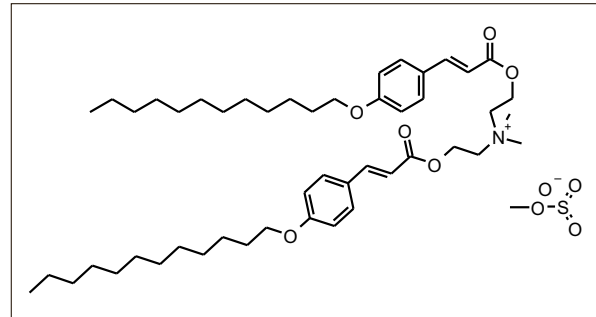

Fig. 11. Liposomogenic substance 101a: bis\{2-[3-(-dodecyloxyphenyl)acryloyloxy]ethyl\} dimethylammonium methosulphate.
Liposomes were prepared via solvent injection, a technique used for the preparation of small unilamellar vesicles. The liposomogenic substance is dissolved in ethanol, and the solution is slowly injected into a surplus of water at a temperature above the melting temperature of the amphiphile. After injection, the non-aqueous solvent can be removed by solvent exchange via diafiltration. The procedures are described in example 10 of ref. [13]. The existence of liposomes was verified using multi-angle static and dynamic light scattering as described in ref. [14]. An average diameter of $63 \mathrm{~nm}$ was obtained.

For the assessment of water-resistance of the liposomogenic UV absorber, the horny layer of the skin from pig ears was prepared as described in example 10 of ref. [13]. The measurements took place in a Franz-type static diffusion cell, filled with the liposome preparation.

Analysis of the amount of UV absorber adsorbed on the skin was performed using UV spectroscopy. Fig. 12 shows results of an experiment with liposomes of compound 101a in comparison with phenyl benzimidazole sulfonic acid (PBSA), a common water soluble UV absorber used in sunscreens.

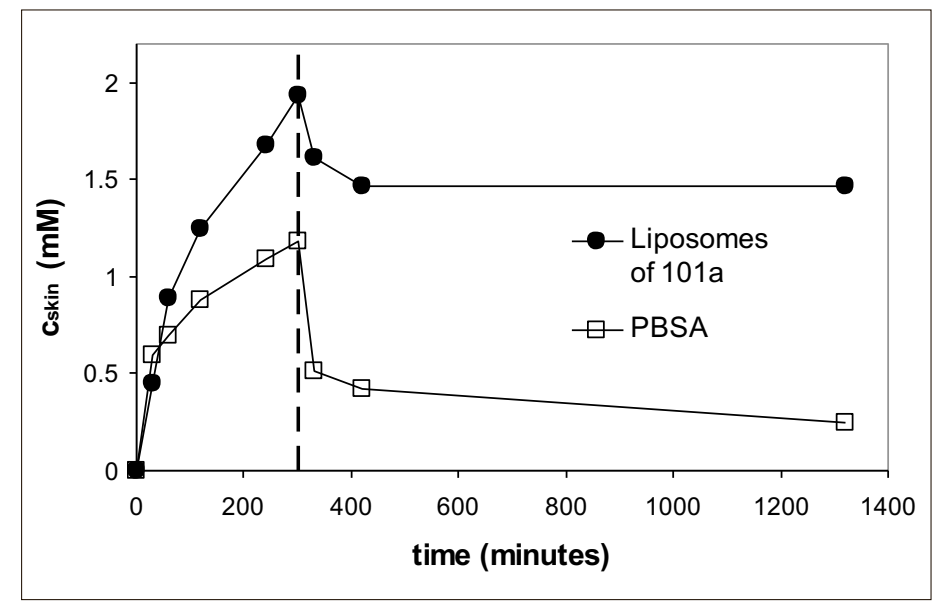

Fig. 12. Adsorption/ desorption experiment with liposomes of compound 101a (c $=10 \mathrm{mM}$ ) and an aqueous solution of PBSA (also at $\mathrm{c}=$ $10 \mathrm{mM})$.

Table 1. Results of adsorption/desorption experiment with 101a and PBSA.

\begin{tabular}{|l|l|l|l|}
\hline UV Absorber & $\begin{array}{l}\mathrm{c}_{\text {skin }}(\mathrm{mM}) \text { after } \\
\text { adsorption }(5 \mathrm{~h}) \\
\text { from 10 } \mathrm{mM} \text { solution } \\
\text { of the liposome } \\
\text { suspension }\end{array}$ & $\begin{array}{l}\mathrm{c}_{\text {skin }}(\mathrm{mM}) \\
\text { after desorption } \\
(17 \mathrm{~h})\end{array}$ & water resistance \\
\hline $\begin{array}{l}\text { 101a, liposome } \\
\text { preparation }\end{array}$ & 1.9 & 1.5 & $79 \%$ \\
\hline $\begin{array}{l}\text { PBSA, aqueous } \\
\text { solution }\end{array}$ & 1.2 & 0.3 & $25 \%$ \\
\hline
\end{tabular}


This approach was discontinued not for technical but for commercial reasons: it was found that the cost of production of such a liposomogenic UV absorber would be prohibitively high, preventing its use in sunscreen formulations.

\section{Conclusion}

Triggered by new requirements towards better UV protection, BEMT (Tinosorb ${ }^{\circledR} \mathrm{S}$ ), MBBT (Tinosorb ${ }^{\circledR} \mathrm{M}$ ) and TBPT (Tinosorb ${ }^{\circledR} \mathrm{A} 2 \mathrm{~B}$ ) have been developed as cosmetic UV absorbers. These filters give formulators new possibilities to cover the UV range from 290 to 400 $\mathrm{nm}$, and to use less filter due to superior efficacy. Today, sunscreens of many different brands apply these UV absorbers. The described innovations were only possible through early anticipation of unmet market needs and by combining synthesis research, formulation technology, photophysics, chemical process development and technical marketing. The development of cosmetic UV absorbers has proven to be a demanding task and a long journey, taking up to 10 years from product idea to commercialization.
Is there still space for new developments in cosmetic UV absorbers? The author strongly believes that the answer is yes. For instance, consumers in Asia are increasingly asking for products which prevent or, at least, delay UV-A induced skin tanning. If exposed to sunlight, the skin should remain light and fair. This claim can only be realized by incorporation of highly efficient UV-A 1 absorbers with a maximum extinction at around 380 $\mathrm{nm}$ into the sunscreen formulation. Future research will reveal if the use of cosmetic UV absorbers can be extended from skin protection to skin tanning prevention, and beyond - claims which are not even known today.

\section{Acknowledgements}

The author thanks Dr. Bernd Herzog for the spectroscopic measurements, physico-chemical evaluations and for illustrative application results in this article and for more than 20 years of fruitful cooperation in the fascinating field of cosmetic UV absorbers. Contributions from many other colleagues from the former R\&D team at Ciba-Geigy / Ciba Specialty Chemicals and the present R\&D team at BASF are also gratefully acknowledged.

Received: April 27, 2016
[1] K. F. De Polo, 'A Short Textbook of Cosmetology', Verlag für chemische Industrie, H. Ziolkowsky GmbH, Augsburg, 1998, pp 86-127.

[2] B. Herzog, D. Hüglin, U. Osterwalder, in 'Sunscreens - Regulations and Commercial Development', Ed. N. A. Shaath, Taylor \& Francis, Boca Raton, 2005, pp 291-320.

[3] B. Herzog, D. Hüglin, E. Borsos, A. Stehlin, H. Luther, Chimia 2004, 58, 66

[4] A. Valet, 'Light Stabilizers for Paints', Vincentz, Hannover, 1997, pp 72-121.

[5] H. Zweifel, 'Plastic Additives Handbook', Hanser, Munich, 2001, pp 206-217.

[6] J. E. Otterstedt, J. Phys. Chem. 1973, 58, 5716.

[7] D. Hüglin, B. Herzog, S. Mongiat, 'Hydroxyphenyltriazines: a new generation of cosmetic UV filters with superior photoprotection", Oral Presentation. 22 $2^{\text {nd }}$ IFSCC, Edinburgh, Sep. 23-26, 2002.

[8] Scientific Committee on Consumer Safety of the European Commission (SCCS/1429/11).

[9] B. Herzog, K. Quass, E. Schmidt, S. Müller, H Luther, J. Colloid Interface Sci. 2004, 276, 354.

[10] F. Elbe, J. Keck, A. P. Flügge, H. E. A. Kramer, P. Fischer, P. Hayoz, D. Leppard, G. Rytz, W. Kaim, M. Ketterle, J. Phys. Chem. A 2000, 104, 8296.

[11] B. Herzog, M. Wehrle, K. Quass, Pharma. Photochem. 2009, 85, 869 .

[12] M. Shimomura, R. Ando, T. Kunitake, Ber Bunsenges. Phys. Chem. 1983, 87, 1134.

[13] H. Luther, D. Hüglin, B. Herzog, US Patent 6 015575,2000

[14] B. Herzog, A. Katzenstein, K. Quass, A. Stehlin, H. Luther, J. Colloid Interface Sci. 2004, 271 , 136. 